\title{
Uncertain SEIAR model for COVID-19 cases in China
}

\author{
Lifen Jia ${ }^{1} \cdot$ Wei Chen ${ }^{1}[$ \\ Accepted: 7 September 2020 / Published online: 24 September 2020 \\ (c) Springer Science+Business Media, LLC, part of Springer Nature 2020
}

\begin{abstract}
The Susceptible-Exposed-Infectious-Asymptomatic-Removed (SEIAR) epidemic model is one of most frequently used epidemic models. As an application of uncertain differential equations to epidemiology, an uncertain SEIAR model is derived which considers the human uncertainty factors during the spread of an epidemic. The parameters in the uncertain epidemic model are estimated with the numbers of COVID-19 cases in China, and a prediction to the possible numbers of active cases is made based on the estimates.
\end{abstract}

Keywords Uncertainty theory · Uncertain differential equation · Uncertain SEIAR model $\cdot$ COVID-19 $\cdot$ Parameter estimation

\section{Introduction}

Epidemics have always been a threat to human health. To describe and predict the spread of an epidemic, various models have been built such as the SIS model, SIR model, SEIR model and SEIAR model. Since the establishment of stochastic differential equation theory in 1950s, stochastic epidemic models have been investigated for the reasons of indeterminate factors during the spread process of an epidemic. For example, Gray et al. (2011) presented a stochastic SIS model, and gave some conditions for extinction and persistence of the disease. Ji et al. (2012) discussed a stochastic SIR model, and proved the stability conditions of disease-free equilibrium. Artalejo et al. (2015) presented a stochastic SEIR model, and studied the evolution of the epidemic before its extinction. As we know, stochastic differential equations are applicable to dynamic systems with random factors rather than human uncertainty, so it is questionable whether they can properly describe epidemic systems which are heavily affected by human behaviors.

$\otimes$ Wei Chen

chenwei@cueb.edu.cn

1 School of Management and Engineering, Capital University of Economics and Business, Beijing, China 
As a branch of mathematics for modelling human uncertainty, the uncertainty theory was founded by Liu (2007) and perfected by Liu (2009). Within the framework of uncertainty theory, the concept of uncertain differential equation was proposed by Liu (2008) to describe dynamic systems with human uncertainty. Chen and Liu (2010) gave a sufficient condition for an uncertain differential equation to have a unique solution, and Yao et al. (2013) proved some stability theorems about uncertain differential equations. The structure of the solution of an uncertain differential equation was found by Yao and Chen (2013), based on which various numerical methods have been designed to solve uncertain differential equations, such as Yang and Ralescu (2015), Gao (2016), and Zhang et al. (2017). In order to estimate the parameters in uncertain differential equations based on observed data, Yao and Liu (2020) presented the method of moments, which was extended to the generalized method of moments by Liu (2020). In addition, the least squares estimation and the maximum likelihood estimation for uncertain differential equations were presented by Sheng et al. (2020) and Liu and Liu (2020), respectively.

As an application of uncertain differential equations to finance, uncertain finance theory was extended during the past years. For example, Liu (2009) assumed the price of a stock follows a lognormal uncertainty distribution, and derived the European option pricing formulas. Chen and Gao (2013) described the short-term interest rate with uncertain differential equations, and investigated the pricing problems of zerocoupon bonds. Uncertain differential equations have also been applied to optimal control (Zhu 2010), game theory (Yang and Gao 2015), population growth model (Sheng et al. 2017) and pharmacokinetics model (Liu and Yang 2020), etc.

The investigation of uncertain epidemic models was initialized by Li et al. (2017), where an uncertain SIS model was constructed and the disease-free equilibrium was discussed. After that, the uncertain SIS model was generalized to the cases with standard incidence and demography (Fang et al. 2018) and with nonlinear incidence and demography (Li and Teng 2019). However, applications of these SIS models are limited because the groups of exposed individuals and recovered individuals with potential immunity are not taken into consideration in these models. In this paper, we aim to build a more general epidemic model by means of uncertain differential equations that is called uncertain SEIAR model. Groups of susceptible individuals, exposed individuals, symptomatically infected individuals, asymptomatically infected individuals, and removed individuals are all considered in such a model, and its application to the COVID-19 cases in China is also provided. The rest of this paper is organized as follows. Section 2 introduces some basic concepts related to uncertain differential equations. Section 3 derives the uncertain SEIAR model, and gives the uncertain SEIR model and the uncertain SIR model as degenerated forms of uncertain SEIAR model. Section 4 estimates the parameters in the uncertain epidemic model by using the numbers of COVID-19 cases in China, and Sect. 5 predicts the possible numbers of COVID-19 active cases in China by means of the uncertain epidemic model with those estimated parameters. Finally, some conclusion are made in Sect. 6. 


\section{Preliminary}

The uncertain measure $\mathcal{M}$ is a set function from a measurable space $(\Gamma, \mathcal{L})$ to the interval $[0,1]$ which satisfies the normality, duality, subadditivity and product axioms. An uncertain variable $\xi$ is a measurable function from the uncertainty space $(\Gamma, \mathcal{L}, \mathcal{M})$ to the set of real numbers.

Definition 1 (Liu 2007) The uncertainty distribution $\Phi$ of an uncertain variable $\xi$ is defined by

$$
\Phi(x)=\mathcal{M}\{\xi \leq x\}
$$

for any real number $x$.

An uncertain variable $\xi$ is called normal if it has an uncertainty distribution

$$
\Phi(x)=\left(1+\exp \left(\frac{\pi(e-x)}{\sqrt{3} \sigma}\right)\right)^{-1}, \quad x \in \Re
$$

denoted by $\mathcal{N}(e, \sigma)$. A normal uncertainty distribution is called standard if $e=0$ and $\sigma=1$.

Definition 2 (Liu 2007) Let $\xi$ be an uncertain variable, and let $k$ be a positive integer. Then the $k$-th moment of $\xi$ is

$$
E\left[\xi^{k}\right]=\int_{0}^{+\infty} \mathcal{M}\left\{\xi^{k} \geq x\right\} \mathrm{d} x-\int_{-\infty}^{0} \mathcal{M}\left\{\xi^{k} \leq x\right\} \mathrm{d} x
$$

provided that at least one of the two integrals is finite.

The first and second moments of a standard normal uncertain variable $\mathcal{N}(0,1)$ are 0 and 1 , respectively.

Definition 3 (Liu 2009) An uncertain process $C_{t}$ is said to be a Liu process if

(i) $C_{0}=0$ and almost all sample paths are Lipschitz continuous;

(ii) $C_{t}$ has stationary and independent increments;

(iii) every increment $C_{s+t}-C_{s}$ is a normal uncertain variable $\mathcal{N}(0, t)$.

Definition 4 (Liu 2009) Let $X_{t}$ be an uncertain process, and let $C_{t}$ be a Liu process. For any partition of closed interval $[a, b]$ with $a=t_{1}<t_{2}<\cdots<t_{k+1}=b$, the mesh is written as

$$
\Delta=\max _{1 \leq i \leq k}\left|t_{i+1}-t_{i}\right|
$$

Then Liu integral of $X_{t}$ with respect to $C_{t}$ is defined as

$$
\int_{a}^{b} X_{t} \mathrm{~d} C_{t}=\lim _{\Delta \rightarrow 0} \sum_{i=1}^{k} X_{t_{i}} \cdot\left(C_{t_{i+1}}-C_{t_{i}}\right)
$$


provided that the limit exists almost surely and is finite.

Definition 5 (Liu 2008) Suppose that $C_{t}$ is a Liu process, and $f$ and $g$ are two measurable functions. Then

$$
\mathrm{d} X_{t}=f\left(t, X_{t}\right) \mathrm{d} t+g\left(t, X_{t}\right) \mathrm{d} C_{t}
$$

is called an uncertain differential equation.

The integral form of the uncertain differential equation (1) is

$$
X_{t}=X_{0}+\int_{0}^{t} f\left(s, X_{s}\right) \mathrm{d} s+\int_{0}^{t} g\left(s, X_{s}\right) \mathrm{d} C_{s} .
$$

\section{Uncertain SEIAR model}

In an SEIAR model, the population is divided into 5 groups, namely, susceptible individuals, exposed individuals, symptomatically infected individuals, asymptomatically infected individuals, and removed individuals (recovered or dead). Susceptible individuals will become exposed individuals after contact with symptomatically infected individuals or asymptomatically infected individuals; Exposed individuals will possibly become symptomatically infected individuals or asymptomatically infected individuals; Symptomatically infected individuals and asymptomatically infected individuals will be removed in some proportions; Removed individuals will not become susceptible individuals again. For simplicity, the numbers of individuals in these 5 groups at the time $t$ are denoted by $S_{t}, E_{t}, I_{t}, A_{t}$ and $R_{t}$ in the uncertain SEIAR model, respectively.

\subsection{Equation of susceptible individuals}

Let $\beta_{1 t}$ denote the contact rate between a susceptible individual and a symptomatically infected individual, and $\beta_{2 t}$ denote the contact rate between a susceptible individual and an asymptomatically infected individual. Considering the human uncertainty during the contact process, we assume

$$
\beta_{1 t}=\beta_{I}+\sigma_{1} \cdot \text { "Noise" }
$$

where $\beta_{I}$ is a nonnegative number, and "Noise" is a normal uncertain variable $\mathcal{N}(0,1)$. Representing the "Noise" by

$$
\frac{C_{1, t+\Delta t}-C_{1 t}}{\Delta t}
$$

where $C_{1 t}$ is a Liu process, we further have

$$
\beta_{1 t}=\beta_{I}+\sigma_{1} \frac{C_{1, t+\Delta t}-C_{1 t}}{\Delta t} .
$$


Similarly, the contact rate $\beta_{2 t}$ can be represented as

$$
\beta_{2 t}=\beta_{A}+\sigma_{2} \frac{C_{2, t+\Delta t}-C_{2 t}}{\Delta t}
$$

where $C_{2 t}$ is a Liu process, and $\beta_{A}$ and $\sigma_{2}$ are two nonnegative numbers. Then the increment of the number of susceptible individuals during an infinitesimal time interval $[t, t+\Delta t]$ is

$$
\begin{aligned}
S_{t+\Delta t}-S_{t}= & -\left(\beta_{1 t} S_{t} I_{t} \Delta t+\beta_{2 t} S_{t} A_{t} \Delta t\right) \\
= & -\left(\beta_{I} S_{t} I_{t}+\beta_{A} S_{t} A_{t}\right) \Delta t-\sigma_{1} S_{t} I_{t}\left(C_{1, t+\Delta t}-C_{1 t}\right) \\
& -\sigma_{2} S_{t} A_{t}\left(C_{2, t+\Delta t}-C_{2 t}\right) .
\end{aligned}
$$

For a time interval $[0, t]$ with a partition $0=t_{0}<t_{1}<t_{2}<\cdots<t_{n}=t$, the increment of the number of susceptible individuals during such an interval is

$$
\begin{aligned}
S_{t}-S_{0}= & \sum_{i=0}^{n-1}\left(S_{t_{i+1}}-S_{t_{i}}\right) \\
= & -\sum_{i=0}^{n-1}\left(\beta_{I} S_{t_{i}} I_{t_{i}}+\beta_{A} S_{t_{i}} A_{t_{i}}\right)\left(t_{i+1}-t_{i}\right)-\sum_{i=0}^{n-1} \sigma_{1} S_{t_{i}} I_{t_{i}}\left(C_{1 t_{i+1}}-C_{1 t_{i}}\right) \\
& -\sum_{i=0}^{n-1} \sigma_{2} S_{t_{i}} A_{t_{i}}\left(C_{2 t_{i+1}}-C_{2 t_{i}}\right) \\
\rightarrow & -\int_{0}^{t}\left(\beta_{I} S_{\tau} I_{\tau}+\beta_{A} S_{\tau} A_{\tau}\right) \mathrm{d} \tau-\sigma_{1} \int_{0}^{t} S_{\tau} I_{\tau} \mathrm{d} C_{1 \tau}-\sigma_{2} \int_{0}^{t} S_{\tau} A_{\tau} \mathrm{d} C_{2 \tau}
\end{aligned}
$$

as

$$
\max _{0 \leq i \leq n-1}\left|t_{i+1}-t_{i}\right| \rightarrow 0
$$

The above integral equation can be rewritten as an uncertain differential equation

$$
\begin{aligned}
\mathrm{d} S_{t}= & -\left(\beta_{I} S_{t} I_{t}+\beta_{A} S_{t} A_{t}\right) \mathrm{d} t-\sigma_{1} S_{t} I_{t} \mathrm{~d} C_{1 t} \\
& -\sigma_{2} S_{t} A_{t} \mathrm{~d} C_{2 t} .
\end{aligned}
$$

\subsection{Equation of exposed individuals}

Let $v_{1 t}$ denote the rate that exposed individuals become symptomatically infected individuals, and $v_{2 t}$ denote the rate that exposed individuals become asymptomatically infected individuals. Similar to the assumptions about the contact rates in Sect. 3.1, we assume that

$$
v_{1 t}=v_{I}+\sigma_{3} \frac{C_{3, t+\Delta t}-C_{3 t}}{\Delta t}
$$


and

$$
v_{2 t}=v_{A}+\sigma_{4} \frac{C_{4, t+\Delta t}-C_{4 t}}{\Delta t}
$$

where $C_{3 t}$ and $C_{4 t}$ are two Liu processes, and $v_{I}, v_{A}, \sigma_{3}$ and $\sigma_{4}$ are some nonnegative numbers. Then the increment of the number of exposed individuals during an infinitesimal time interval $[t, t+\Delta t]$ is

$$
\begin{aligned}
E_{t+\Delta t}-E_{t}= & \left(S_{t}-S_{t+\Delta t}\right)-\left(v_{1 t}+v_{2 t}\right) E_{t} \Delta t \\
= & \left(S_{t}-S_{t+\Delta t}\right)-\left(v_{I}+v_{A}\right) E_{t} \Delta t-\sigma_{3} E_{t}\left(C_{3, t+\Delta t}-C_{3 t}\right) \\
& -\sigma_{4} E_{t}\left(C_{4, t+\Delta t}-C_{4 t}\right) .
\end{aligned}
$$

For a time interval $[0, t]$ with a partition $0=t_{0}<t_{1}<t_{2}<\cdots<t_{n}=t$, the increment of the number of exposed individuals during such an interval is

$$
\begin{aligned}
E_{t}-E_{0}= & \sum_{i=0}^{n-1}\left(E_{t_{i+1}}-E_{t_{i}}\right) \\
= & S_{0}-S_{t}-\sum_{i=0}^{n-1}\left(v_{I}+v_{A}\right) E_{t_{i}}\left(t_{i+1}-t_{i}\right)-\sum_{i=0}^{n-1} \sigma_{3} E_{t_{i}}\left(C_{3 t_{i+1}}-C_{3 t_{i}}\right) \\
& -\sum_{i=0}^{n-1} \sigma_{4} E_{t_{i}}\left(C_{4 t_{i+1}}-C_{4 t_{i}}\right) \\
\rightarrow & \int_{0}^{t}\left(\beta_{I} S_{\tau} I_{\tau}+\beta_{A} S_{\tau} A_{\tau}\right) \mathrm{d} \tau+\sigma_{1} \int_{0}^{t} S_{\tau} I_{\tau} \mathrm{d} C_{1 \tau}+\sigma_{2} \int_{0}^{t} S_{\tau} A_{\tau} \mathrm{d} C_{2 \tau} \\
& -\left(v_{I}+v_{A}\right) \int_{0}^{t} E_{\tau} \mathrm{d} \tau-\sigma_{3} \int_{0}^{t} E_{\tau} \mathrm{d} C_{3 \tau}-\sigma_{4} \int_{0}^{t} E_{\tau} \mathrm{d} C_{4 \tau}
\end{aligned}
$$

as

$$
\max _{0 \leq i \leq n-1}\left|t_{i+1}-t_{i}\right| \rightarrow 0
$$

The above integral equation can be rewritten as an uncertain differential equation

$$
\begin{aligned}
\mathrm{d} E_{t}= & \left(\beta_{I} S_{t} I_{t}+\beta_{A} S_{t} A_{t}-v_{I} E_{t}-v_{A} E_{t}\right) \mathrm{d} t+\sigma_{1} S_{t} I_{t} \mathrm{~d} C_{1 t}+\sigma_{2} S_{t} A_{t} \mathrm{~d} C_{2 t} \\
& -\sigma_{3} E_{t} \mathrm{~d} C_{3 t}-\sigma_{4} E_{t} \mathrm{~d} C_{4 t} .
\end{aligned}
$$

\subsection{Equation of symptomatically infected individuals}

Let $v_{1 t}$ denote the rate that exposed individuals become symptomatically infected individuals, and $\mu_{1 t}$ denote the removed (recovered or dead) rate of symptomatically 
infected individuals. As the assumption in Sect. 3.2, we have

$$
v_{1 t}=v_{I}+\sigma_{3} \frac{C_{3, t+\Delta t}-C_{3 t}}{\Delta t} .
$$

Similarly, we assume that

$$
\mu_{1 t}=\mu_{I}+\sigma_{5} \frac{C_{5, t+\Delta t}-C_{5 t}}{\Delta t}
$$

where $C_{5 t}$ is a Liu process, and $\mu_{I}$ and $\sigma_{5}$ are two nonnegative numbers. Then the increment of the number of symptomatically infected individuals during an infinitesimal time interval $[t, t+\Delta t]$ is

$$
\begin{aligned}
I_{t+\Delta t}-I_{t} & =v_{1 t} E_{t} \Delta t-\mu_{1 t} I_{t} \Delta t \\
& =\left(v_{I} E_{t}-\mu_{I} I_{t}\right) \Delta t+\sigma_{3} E_{t}\left(C_{3, t+\Delta t}-C_{3 t}\right)-\sigma_{5} I_{t}\left(C_{5, t+\Delta t}-C_{5 t}\right) .
\end{aligned}
$$

For a time interval $[0, t]$ with a partition $0=t_{0}<t_{1}<t_{2}<\cdots<t_{n}=t$, the increment of the number of symptomatically infected individuals during such an interval is

$$
\begin{aligned}
I_{t}-I_{0}= & \sum_{i=0}^{n-1}\left(I_{t_{i+1}}-I_{t_{i}}\right) \\
= & \sum_{i=0}^{n-1}\left(v_{I} E_{t_{i}}-\mu_{I} I_{t_{i}}\right)\left(t_{i+1}-t_{i}\right)+\sum_{i=0}^{n-1} \sigma_{3} E_{t_{i}}\left(C_{3 t_{i+1}}-C_{3 t_{i}}\right) \\
& -\sum_{i=0}^{n-1} \sigma_{5} I_{t_{i}}\left(C_{5 t_{i+1}}-C_{5 t_{i}}\right) \\
& \rightarrow \int_{0}^{t}\left(v_{I} E_{\tau}-\mu_{I} I_{\tau}\right) \mathrm{d} \tau+\sigma_{3} \int_{0}^{t} E_{\tau} \mathrm{d} C_{3 \tau}-\sigma_{5} \int_{0}^{t} I_{\tau} \mathrm{d} C_{5 \tau}
\end{aligned}
$$

as

$$
\max _{0 \leq i \leq n-1}\left|t_{i+1}-t_{i}\right| \rightarrow 0
$$

The above integral equation can be rewritten as an uncertain differential equation

$$
\mathrm{d} I_{t}=\left(v_{I} E_{t}-\mu_{I} I_{t}\right) \mathrm{d} t+\sigma_{3} E_{t} \mathrm{~d} C_{3 t}-\sigma_{5} I_{t} \mathrm{~d} C_{5 t}
$$

\subsection{Equation of asymptomatically infected individuals}

Let $v_{2 t}$ denote the rate that exposed individuals become asymptomatically infected individuals, and $\mu_{2 t}$ denote the removed (recovered or dead) rate of asymptomatically 
infected individuals. As the assumption in Sect. 3.2, we have

$$
v_{2 t}=v_{A}+\sigma_{4} \frac{C_{4, t+\Delta t}-C_{4 t}}{\Delta t} .
$$

Similarly, we assume that

$$
\mu_{2 t}=\mu_{A}+\sigma_{6} \frac{C_{6, t+\Delta t}-C_{6 t}}{\Delta t}
$$

where $C_{6 t}$ is a Liu process, and $\mu_{A}$ and $\sigma_{6}$ are two nonnegative numbers. Then the increment of the number of asymptomatically infected individuals during an infinitesimal time interval $[t, t+\Delta t]$ is

$$
\begin{aligned}
A_{t+\Delta t}-A_{t} & =v_{2 t} E_{t} \Delta t-\mu_{2 t} A_{t} \Delta t \\
& =\left(v_{A} E_{t}-\mu_{A} A_{t}\right) \Delta t+\sigma_{4} E_{t}\left(C_{4, t+\Delta t}-C_{4 t}\right)-\sigma_{6} A_{t}\left(C_{6, t+\Delta t}-C_{6 t}\right) .
\end{aligned}
$$

For a time interval $[0, t]$ with a partition $0=t_{0}<t_{1}<t_{2}<\cdots<t_{n}=t$, the increment of the number of asymptomatically infected individuals during such an interval is

$$
\begin{aligned}
A_{t}-A_{0}= & \sum_{i=0}^{n-1}\left(A_{t_{i+1}}-A_{t_{i}}\right) \\
= & \sum_{i=0}^{n-1}\left(v_{A} E_{t_{i}}-\mu_{A} A_{t_{i}}\right)\left(t_{i+1}-t_{i}\right)+\sum_{i=0}^{n-1} \sigma_{4} E_{t_{i}}\left(C_{4 t_{i+1}}-C_{4 t_{i}}\right) \\
& -\sum_{i=0}^{n-1} \sigma_{6} A_{t_{i}}\left(C_{6 t_{i+1}}-C_{6 t_{i}}\right) \\
\rightarrow & \int_{0}^{t}\left(v_{A} E_{\tau}-\mu_{A} A_{\tau}\right) \mathrm{d} \tau+\sigma_{4} \int_{0}^{t} E_{\tau} \mathrm{d} C_{4 \tau}-\sigma_{6} \int_{0}^{t} A_{\tau} \mathrm{d} C_{6 \tau}
\end{aligned}
$$

as

$$
\max _{0 \leq i \leq n-1}\left|t_{i+1}-t_{i}\right| \rightarrow 0
$$

The above integral equation can be rewritten as an uncertain differential equation

$$
\mathrm{d} A_{t}=\left(v_{A} E_{t}-\mu_{A} A_{t}\right) \mathrm{d} t+\sigma_{4} E_{t} \mathrm{~d} C_{4 t}-\sigma_{6} A_{t} \mathrm{~d} C_{6 t}
$$

\subsection{Equation of removed individuals}

Let $\mu_{1 t}$ denote the removed rate of symptomatically infected individuals, and $\mu_{2 t}$ denote the removed rate of asymptomatically infected individuals. As the assumptions 
in Sects. 3.3 and 3.4, we have

$$
\mu_{1 t}=\mu_{I}+\sigma_{5} \frac{C_{5, t+\Delta t}-C_{5 t}}{\Delta t}
$$

and

$$
\mu_{2 t}=\mu_{A}+\sigma_{6} \frac{C_{6, t+\Delta t}-C_{6 t}}{\Delta t}
$$

The increment of the number of removed individuals during an infinitesimal time interval $[t, t+\Delta t]$ is

$$
\begin{aligned}
R_{t+\Delta t}-R_{t} & =\mu_{1 t} I_{t} \Delta t+\mu_{2 t} A_{t} \Delta t \\
& =\left(\mu_{I} I_{t}+\mu_{A} A_{t}\right) \Delta t+\sigma_{5} I_{t}\left(C_{5, t+\Delta t}-C_{5 t}\right)+\sigma_{6} A_{t}\left(C_{6, t+\Delta t}-C_{6 t}\right) .
\end{aligned}
$$

For a time interval $[0, t]$ with a partition $0=t_{0}<t_{1}<t_{2}<\cdots<t_{n}=t$, the increment of the number of removed individuals during such an interval is

$$
\begin{aligned}
R_{t}-R_{0}= & \sum_{i=0}^{n-1}\left(R_{t_{i+1}}-R_{t_{i}}\right) \\
= & \sum_{i=0}^{n-1}\left(\mu_{I} I_{t_{i}}+\mu_{A} A_{t_{i}}\right)\left(t_{i+1}-t_{i}\right)+\sum_{i=0}^{n-1} \sigma_{5} I_{t_{i}}\left(C_{5 t_{i+1}}-C_{5 t_{i}}\right) \\
& +\sum_{i=0}^{n-1} \sigma_{6} A_{t_{i}}\left(C_{6 t_{i+1}}-C_{6 t_{i}}\right) \\
& \rightarrow \int_{0}^{t}\left(\mu_{I} I_{\tau}+\mu_{A} A_{\tau}\right) \mathrm{d} \tau+\sigma_{5} \int_{0}^{t} I_{\tau} \mathrm{d} C_{5 \tau}+\sigma_{6} \int_{0}^{t} A_{\tau} \mathrm{d} C_{6 \tau}
\end{aligned}
$$

as

$$
\max _{0 \leq i \leq n-1}\left|t_{i+1}-t_{i}\right| \rightarrow 0
$$

The above integral equation can be rewritten as an uncertain differential equation

$$
\mathrm{d} R_{t}=\left(\mu_{I} I_{t}+\mu_{A} A_{t}\right) \mathrm{d} t+\sigma_{5} I_{t} \mathrm{~d} C_{5 t}+\sigma_{6} A_{t} \mathrm{~d} C_{6 t} .
$$

\subsection{Uncertain SEIAR model}

Based on the equations in Sects. 3.1-3.5, we propose the uncertain SEIAR model 


$$
\left\{\begin{aligned}
\mathrm{d} S_{t}= & -\left(\beta_{I} S_{t} I_{t}+\beta_{A} S_{t} A_{t}\right) \mathrm{d} t-\sigma_{1} S_{t} I_{t} \mathrm{~d} C_{1 t}-\sigma_{2} S_{t} A_{t} \mathrm{~d} C_{2 t} \\
\mathrm{~d} E_{t}= & \left(\beta_{I} S_{t} I_{t}+\beta_{A} S_{t} A_{t}-v_{I} E_{t}-v_{A} E_{t}\right) \mathrm{d} t+\sigma_{1} S_{t} I_{t} \mathrm{~d} C_{1 t} \\
& \quad+\sigma_{2} S_{t} A_{t} \mathrm{~d} C_{2 t}-\sigma_{3} E_{t} \mathrm{~d} C_{3 t}-\sigma_{4} E_{t} \mathrm{~d} C_{4 t} \\
\mathrm{~d} I_{t}= & \left(v_{I} E_{t}-\mu_{I} I_{t}\right) \mathrm{d} t+\sigma_{3} E_{t} \mathrm{~d} C_{3 t}-\sigma_{5} I_{t} \mathrm{~d} C_{5 t} \\
\mathrm{~d} A_{t}= & \left(v_{A} E_{t}-\mu_{A} A_{t}\right) \mathrm{d} t+\sigma_{4} E_{t} \mathrm{~d} C_{4 t}-\sigma_{6} A_{t} \mathrm{~d} C_{6 t} \\
\mathrm{~d} R_{t}= & \left(\mu_{I} I_{t}+\mu_{A} A_{t}\right) \mathrm{d} t+\sigma_{5} I_{t} \mathrm{~d} C_{5 t}+\sigma_{6} A_{t} \mathrm{~d} C_{6 t}
\end{aligned}\right.
$$

to describe an epidemic system with uncertain information, where $S_{t}, E_{t}, I_{t}, A_{t}$ and $R_{t}$ denote the numbers of susceptible individuals, exposed individuals, symptomatically infected individuals, asymptomatically infected individuals, and removed individuals, respectively, $C_{i t}, i=1,2, \ldots, 6$ are Liu processes, $\beta_{I}, \beta_{A}, v_{I}, v_{A}, \mu_{I}, \mu_{A}$ and $\sigma_{i}$, $i=1,2, \ldots, 6$ are some nonnegative numbers.

The uncertain SEIAR model (7) degenerates to an uncertain SEIR model if the group of asymptomatically infected individuals is neglected. Setting $A_{t}=0$ and $v_{A}=\sigma_{4}=0$ in the model (7), we get the uncertain SEIR model

$$
\left\{\begin{array}{l}
\mathrm{d} S_{t}=-\beta_{I} S_{t} I_{t} \mathrm{~d} t-\sigma_{1} S_{t} I_{t} \mathrm{~d} C_{1 t} \\
\mathrm{~d} E_{t}=\left(\beta_{I} S_{t} I_{t}-v_{I} E_{t}\right) \mathrm{d} t+\sigma_{1} S_{t} I_{t} \mathrm{~d} C_{1 t}-\sigma_{3} E_{t} \mathrm{~d} C_{3 t} \\
\mathrm{~d} I_{t}=\left(v_{I} E_{t}-\mu_{I} I_{t}\right) \mathrm{d} t+\sigma_{3} E_{t} \mathrm{~d} C_{3 t}-\sigma_{5} I_{t} \mathrm{~d} C_{5 t} \\
\mathrm{~d} R_{t}=\mu_{I} I_{t} \mathrm{~d} t+\sigma_{5} I_{t} \mathrm{~d} C_{5 t}
\end{array}\right.
$$

Furthermore, the uncertain SEIR model (8) degenerates to an uncertain SIR model if the group of exposed individuals is neglected. In this case, all the exposed individuals are regarded as infected individuals. Replacing $v_{I} E_{t} \mathrm{~d} t+\sigma_{3} E_{t} \mathrm{~d} C_{3 t}$ with $\beta_{I} S_{t} I_{t} \mathrm{~d} t+$ $\sigma_{1} S_{t} I_{t} \mathrm{~d} C_{1 t}$ in the model (8), we get the uncertain SIR model

$$
\left\{\begin{array}{l}
\mathrm{d} S_{t}=-\beta_{I} S_{t} I_{t} \mathrm{~d} t-\sigma_{1} S_{t} I_{t} \mathrm{~d} C_{1 t} \\
\mathrm{~d} I_{t}=\left(\beta_{I} S_{t} I_{t}-\mu_{I} I_{t}\right) \mathrm{d} t+\sigma_{1} S_{t} I_{t} \mathrm{~d} C_{1 t}-\sigma_{5} I_{t} \mathrm{~d} C_{5 t} \\
\mathrm{~d} R_{t}=\mu_{I} I_{t} \mathrm{~d} t+\sigma_{5} I_{t} \mathrm{~d} C_{5 t}
\end{array}\right.
$$

\section{Parameter estimation with COVID-19 cases}

Based on the COVID-19 cases in Mainland China, we estimate the parameters in the uncertain epidemic models in this section. The focus of this research is on the number of active cases, so we perform the parameter estimation with respect to the uncertain SIR model for simplicity. Beside, we accept the following stipulation in order to further simplify the parameter estimation process.

Stipulation: The number of susceptible individuals is a constant that is 1.4 billion. According to National Bureau of Statistics of the People's Republic of China, the population of Mainland China is about 1.40005 billion, while the number of confirmed cases in mainland China is 82,052 as of April 11, 2020. Hence, we stipulate that the number of susceptible individuals is 1.4 billion for simplicity. 
Following the above stipulation, the uncertain SIR model (9) is simplified to an IR model

$$
\left\{\begin{array}{l}
\mathrm{d} I_{t}=\left(\beta_{I} S_{0} I_{t}-\mu_{I} I_{t}\right) \mathrm{d} t+\sigma_{1} S_{0} I_{t} \mathrm{~d} C_{1 t}-\sigma_{5} I_{t} \mathrm{~d} C_{5 t} \\
\mathrm{~d} R_{t}=\mu_{I} I_{t} \mathrm{~d} t+\sigma_{5} I_{t} \mathrm{~d} C_{5 t}
\end{array}\right.
$$

where $S_{0}=1.4$ billion is the number of susceptible individuals, $I_{t}$ and $R_{t}$ denote the numbers of symptomatically infected individuals and removed individuals, respectively, $C_{1 t}$ and $C_{5 t}$ are two Liu processes which are assumed to be independent for the purpose of parameter estimation, and $\beta_{I}, \mu_{I}, \sigma_{1}$ and $\sigma_{5}$ are nonnegative parameters to be estimated.

The following parameter estimation process mainly follows the method of moments for uncertain differential equations by Yao and Liu (2020) and for multi-factor uncertain differential equations by Liu and Yang (2020). Consider the equation of removed individuals

$$
\mathrm{d} R_{t}=\mu_{I} I_{t} \mathrm{~d} t+\sigma_{5} I_{t} \mathrm{~d} C_{5 t}
$$

in the uncertain IR model (10). A Euler difference scheme of the uncertain differential equation (11) is

$$
R_{t_{i+1}}-R_{t_{i}}=\mu_{I} I_{t_{i}}\left(t_{i+1}-t_{i}\right)+\sigma_{5} I_{t_{i}}\left(C_{5 t_{i+1}}-C_{5 t_{i}}\right)
$$

which can be rewritten as

$$
\frac{R_{t_{i+1}}-R_{t_{i}}-\mu_{I} I_{t_{i}}\left(t_{i+1}-t_{i}\right)}{\sigma_{5} I_{t_{i}}\left(t_{i+1}-t_{i}\right)}=\frac{C_{5 t_{i+1}}-C_{5 t_{i}}}{t_{i+1}-t_{i}} .
$$

According to the definition of Liu process, the right expression

$$
\frac{C_{5 t_{i+1}}-C_{5 t_{i}}}{t_{i+1}-t_{i}}
$$

is a standard normal uncertain variable $\mathcal{N}(0,1)$. Hence, when $R_{t_{i}}$ and $I_{t_{i}}$ are assigned the numbers of closed cases and active cases on the $i$-th day, respectively, the left expression

$$
\frac{R_{t_{i+1}}-R_{t_{i}}-\mu_{I} I_{t_{i}}\left(t_{i+1}-t_{i}\right)}{\sigma_{5} I_{t_{i}}\left(t_{i+1}-t_{i}\right)}
$$

can be regarded as samples of a standard normal uncertain variable $\mathcal{N}(0,1)$. Note that the first and second moments of a standard normal uncertain variable $\mathcal{N}(0,1)$ are 0 and 1 , respectively. The estimates $\mu_{I}^{*}$ and $\sigma_{5}^{*}$ of $\mu_{i}$ and $\sigma_{5}$ solve the system of 
equations

$$
\left\{\begin{array}{l}
\frac{1}{n-1} \sum_{i=1}^{n-1} \frac{R_{t_{i+1}}-R_{t_{i}}-\mu_{I} I_{t_{i}}\left(t_{i+1}-t_{i}\right)}{\sigma_{5} I_{t_{i}}\left(t_{i+1}-t_{i}\right)}=0 \\
\frac{1}{n-1} \sum_{i=1}^{n-1}\left(\frac{R_{t_{i+1}}-R_{t_{i}}-\mu_{I} I_{t_{i}}\left(t_{i+1}-t_{i}\right)}{\sigma_{5} I_{t_{i}}\left(t_{i+1}-t_{i}\right)}\right)^{2}=1 .
\end{array}\right.
$$

That is,

$$
\begin{aligned}
\mu_{I}^{*} & =\frac{1}{n-1} \sum_{i=1}^{n-1} \frac{R_{t_{i+1}}-R_{t_{i}}}{I_{t_{i}}\left(t_{i+1}-t_{i}\right)} \\
\sigma_{5}^{*} & =\left(\frac{1}{n-1} \sum_{i=1}^{n-1}\left(\frac{R_{t_{i+1}}-R_{t_{i}}}{I_{t_{i}}\left(t_{i+1}-t_{i}\right)}-\mu_{I}^{*}\right)^{2}\right)^{1 / 2} .
\end{aligned}
$$

Now consider the equation of symptomatically infected individuals

$$
\mathrm{d} I_{t}=\left(\beta_{I} S_{0} I_{t}-\mu_{I} I_{t}\right) \mathrm{d} t+\sigma_{1} S_{0} I_{t} \mathrm{~d} C_{1 t}-\sigma_{5} I_{t} \mathrm{~d} C_{5 t}
$$

in the uncertain IR model (10). Substituting $\mu_{I}$ and $\sigma_{5}$ with $\mu_{I}^{*}$ and $\sigma_{5}^{*}$ that have been determined in Eqs. (12) and (13), we get

$$
\mathrm{d} I_{t}=\left(\beta_{I} S_{0} I_{t}-\mu_{I}^{*} I_{t}\right) \mathrm{d} t+\sigma_{1} S_{0} I_{t} \mathrm{~d} C_{1 t}-\sigma_{5}^{*} I_{t} \mathrm{~d} C_{5 t} .
$$

A Euler difference scheme of the uncertain differential equation (14) is

$$
\begin{aligned}
I_{t_{i+1}}-I_{t_{i}}= & \left(\beta_{I} S_{0} I_{t_{i}}-\mu_{I}^{*} I_{t_{i}}\right)\left(t_{i+1}-t_{i}\right)+\sigma_{1} S_{0} I_{t_{i}}\left(C_{1 t_{i+1}}-C_{1 t_{i}}\right) \\
& -\sigma_{5}^{*} I_{t_{i}}\left(C_{5 t_{i+1}}-C_{5 t_{i}}\right)
\end{aligned}
$$

which can be rewritten as

$$
\begin{gathered}
\frac{I_{t_{i+1}}-I_{t_{i}}-\left(\beta_{I} S_{0} I_{t_{i}}-\mu_{I}^{*} I_{t_{i}}\right)\left(t_{i+1}-t_{i}\right)}{\left(\sigma_{1} S_{0} I_{t_{i}}+\sigma_{5}^{*} I_{t_{i}}\right)\left(t_{i+1}-t_{i}\right)}=\frac{\sigma_{1} S_{0} I_{t_{i}}}{\sigma_{1} S_{0} I_{t_{i}}+\sigma_{5}^{*} I_{t_{i}}} \\
\frac{C_{1 t_{i+1}}-C_{1 t_{i}}}{t_{i+1}-t_{i}}-\frac{\sigma_{5}^{*} I_{t_{i}}}{\sigma_{1} S_{0} I_{t_{i}}+\sigma_{5}^{*} I_{t_{i}}} \frac{C_{5 t_{i+1}}-C_{5 t_{i}}}{t_{i+1}-t_{i}} .
\end{gathered}
$$

It follows from the independence and definition of Liu processes $C_{1 t}$ and $C_{5 t}$ that

$$
\frac{C_{1 t_{i+1}}-C_{1 t_{i}}}{t_{i+1}-t_{i}}
$$

and

$$
\frac{C_{5 t_{i+1}}-C_{5 t_{i}}}{t_{i+1}-t_{i}}
$$


Table 1 Numbers of active cases of COVID-19 in mainland China from February 12 to April 11, 2020. Source COVID-19 Cases Reports by the National Health Commission of the People's Republic of China National Health Commission of the People's Republic of China (2020)

\begin{tabular}{llllllllll}
\hline 52,526 & 55,748 & 56,873 & 57,416 & 57,934 & 58,016 & 57,805 & 56,303 & 54,965 & 53,284 \\
51,606 & 49,824 & 47,672 & 45,604 & 43,258 & 39,919 & 37,414 & 35,329 & 32,652 & 30,004 \\
27,433 & 25,352 & 23,784 & 22,177 & 20,533 & 19,016 & 17,721 & 16,145 & 14,831 & 13,526 \\
12,094 & 10,734 & 9898 & 8976 & 8056 & 7263 & 6569 & 6013 & 5549 & 5120 \\
4735 & 4287 & 3947 & 3460 & 3128 & 2691 & 2396 & 2161 & 2004 & 1863 \\
1727 & 1562 & 1376 & 1299 & 1242 & 1190 & 1160 & 1116 & 1089 & 1138 \\
\hline
\end{tabular}

are independent standard normal uncertain variables with a common uncertainty distribution $\mathcal{N}(0,1)$. Then according to the operational law of uncertain variables, we have

$$
\begin{gathered}
\frac{\sigma_{1} S_{0} I_{t_{i}}}{\sigma_{1} S_{0} I_{t_{i}}+\sigma_{5}^{*} I_{t_{i}}} \frac{C_{1 t_{i+1}}-C_{1 t_{i}}}{t_{i+1}-t_{i}}-\frac{\sigma_{5}^{*} I_{t_{i}}}{\sigma_{1} S_{0} I_{t_{i}}+\sigma_{5}^{*} I_{t_{i}}} \frac{C_{5 t_{i+1}}-C_{5 t_{i}}}{t_{i+1}-t_{i}} \\
\sim \mathcal{N}\left(0, \frac{\sigma_{1} S_{0} I_{t_{i}}}{\sigma_{1} S_{0} I_{t_{i}}+\sigma_{5}^{*} I_{t_{i}}}+\frac{\sigma_{5}^{*} I_{t_{i}}}{\sigma_{1} S_{0} I_{t_{i}}+\sigma_{5}^{*} I_{t_{i}}}\right)=\mathcal{N}(0,1) .
\end{gathered}
$$

Hence, when $I_{t_{i}}$ are assigned the numbers of active cases on the $i$-th day, the left expression

$$
\frac{I_{t_{i+1}}-I_{t_{i}}-\left(\beta_{I} S_{0} I_{t_{i}}-\mu_{I}^{*} I_{t_{i}}\right)\left(t_{i+1}-t_{i}\right)}{\left(\sigma_{1} S_{0} I_{t_{i}}+\sigma_{5}^{*} I_{t_{i}}\right)\left(t_{i+1}-t_{i}\right)}
$$

can be regarded as samples of a standard normal uncertain variable $\mathcal{N}(0,1)$. Then by means of the method of moments, the estimates $\beta_{I}^{*}$ and $\sigma_{1}^{*}$ of $\beta_{I}$ and $\sigma_{1}$ solve the system of equations

$$
\left\{\begin{array}{l}
\frac{1}{n-1} \sum_{i=1}^{n-1} \frac{I_{t_{i+1}}-I_{t_{i}}-\left(\beta_{I} S_{0} I_{t_{i}}-\mu_{I}^{*} I_{t_{i}}\right)\left(t_{i+1}-t_{i}\right)}{\left(\sigma_{1} S_{0} I_{t_{i}}+\sigma_{5}^{*} I_{t_{i}}\right)\left(t_{i+1}-t_{i}\right)}=0 \\
\frac{1}{n-1} \sum_{i=1}^{n-1}\left(\frac{I_{t_{i+1}}-I_{t_{i}}-\left(\beta_{I} S_{0} I_{t_{i}}-\mu_{I}^{*} I_{t_{i}}\right)\left(t_{i+1}-t_{i}\right)}{\left(\sigma_{1} S_{0} I_{t_{i}}+\sigma_{5}^{*} I_{t_{i}}\right)\left(t_{i+1}-t_{i}\right)}\right)^{2}=1 .
\end{array}\right.
$$

That is,

$$
\begin{gathered}
\beta_{I}^{*}=\frac{1}{S_{0}}\left(\frac{1}{n-1} \sum_{i=1}^{n-1} \frac{I_{t_{i+1}}-I_{t_{i}}}{I_{t_{i}}\left(t_{i+1}-t_{i}\right)}+\mu_{I}^{*}\right), \\
\sigma_{1}^{*}=\frac{1}{S_{0}}\left(\left(\frac{1}{n-1} \sum_{i=1}^{n-1}\left(\frac{I_{t_{i+1}}-I_{t_{i}}}{I_{t_{i}}\left(t_{i+1}-t_{i}\right)}-\left(\beta_{I}^{*} S_{0}-\mu_{I}^{*}\right)\right)^{2}\right)^{1 / 2}-\sigma_{5}^{*}\right) .
\end{gathered}
$$

Now, we estimate the parameters $\beta_{I}, \sigma_{1}, \mu_{I}$ and $\sigma_{5}$ in the uncertain IR model (10) based on the numbers of active cases (Table 1) and closed cases (Table 2) in 
Table 2 Numbers of closed cases of COVID-19 in mainland China from February 12 to April 11, 2020. Source COVID-19 Cases Reports by the National Health Commission of the People's Republic of China National Health Commission of the People's Republic of China (2020)

\begin{tabular}{llllllllll}
\hline 7278 & 8103 & 9619 & 11,084 & 12,614 & 14,420 & 16,380 & 18,273 & 20,500 & 23,004 \\
25,330 & 27,326 & 29,986 & 32,460 & 35,239 & 38,905 & 41,837 & 44,495 & 47,374 & 50,147 \\
52,837 & 55,057 & 56,768 & 58,474 & 60,162 & 61,719 & 63,033 & 64,633 & 65,962 & 67,287 \\
68,730 & 70,110 & 70,962 & 71,905 & 72,838 & 73,665 & 74,398 & 74,995 & 75,505 & 75,973 \\
76,436 & 76,931 & 77,338 & 77,880 & 78,266 & 78,748 & 79,074 & 79,357 & 79,550 & 79,726 \\
79,893 & 80,077 & 80,293 & 80,409 & 80,498 & 80,612 & 80,705 & 80,791 & 80,864 & 80,914 \\
\hline
\end{tabular}

Mainland China from February 12 to April 11, 2020, which are released in the COVID19 cases reports by National Health Commission of the People's Republic of China National Health Commission of the People's Republic of China (2020). Details about the computation procedure are given as below.

Step 1 Set $S_{0}=1.4 \times 10^{9}$ and $n=60$.

Step 2 Set February 12 as the first day, February 13 as the second day, and so forth. That is, $t_{i}=i$, and $I_{t_{i}}$ and $R_{t_{i}}$ are the $i$-th numbers in Tables 1 and 2, respectively, for $i=1,2, \cdots, 60$. For example, $I_{t_{3}}=56873$ and $R_{t_{5}}=$ 12614.

Step 3 Compute $\mu_{I}^{*}$ based on Eq. (12).

Step 4 Compute $\sigma_{5}^{*}$ based on Eq. (13).

Step 5 Compute $\beta_{I}^{*}$ based on Eq. (15).

Step 6 Compute $\sigma_{1}^{*}$ based on Eq. (16).

Following the above procedure, we get the estimates of the parameters $\beta_{I}, \sigma_{1}, \mu_{I}$ and $\sigma_{5}$ in the uncertain IR model (10) that are

$$
\beta_{I}^{*}=1.1745 \times 10^{-11}, \quad \sigma_{1}^{*}=7.7866 \times 10^{-12}, \quad \mu_{I}^{*}=0.0785, \quad \sigma_{5}^{*}=0.0296
$$

Hence, the uncertain IR model for COVID-19 in Mainland China from February 12 to April 11, 2020 is

$$
\left\{\begin{array}{l}
\mathrm{d} I_{t}=-0.0620 \cdot I_{t} \mathrm{~d} t+0.0109 \cdot I_{t} \mathrm{~d} C_{1 t}-0.0296 \cdot I_{t} \mathrm{~d} C_{5 t} \\
\mathrm{~d} R_{t}=0.0785 \cdot I_{t} \mathrm{~d} t+0.0296 \cdot I_{t} \mathrm{~d} C_{5 t}
\end{array}\right.
$$

\section{Prediction of the confirmed cases}

Based on the uncertain IR model (10) with the estimated parameters, we predict the possible numbers of active cases $\left(I_{t}\right)$ in this section.

Consider the equation of symptomatically infected individuals

$$
\mathrm{d} I_{t}=\left(\beta_{I}^{*} S_{0} I_{t}-\mu_{I}^{*} I_{t}\right) \mathrm{d} t+\sigma_{1}^{*} S_{0} I_{t} \mathrm{~d} C_{1 t}-\sigma_{5}^{*} I_{t} \mathrm{~d} C_{5 t}
$$


which has a solution

$$
I_{t}=I_{0} \exp \left(\left(\beta_{I}^{*} S_{0}-\mu_{I}^{*}\right) t+\sigma_{1}^{*} S_{0} C_{1 t}-\sigma_{5}^{*} C_{5 t}\right)
$$

Please note that its uncertainty distribution is

$$
\Phi_{t}(x)=\mathcal{M}\left\{I_{t} \leq x\right\}=\mathcal{M}\left\{\sigma_{1}^{*} S_{0} C_{1 t}-\sigma_{5}^{*} C_{5 t} \leq \ln x-\left(\beta_{I}^{*} S_{0}-\mu_{I}^{*}\right) t-\ln I_{0}\right\} .
$$

Since $C_{1 t}$ and $C_{5 t}$ are independent Liu processes with a common uncertainty distribution $\mathcal{N}(0, t)$, we have

$$
\Phi_{t}(x)=\left(1+\exp \left(\frac{\pi\left(\left(\beta_{I}^{*} S_{0}-\mu_{I}^{*}\right) t+\ln I_{0}-\ln x\right)}{\sqrt{3}\left(\sigma_{1}^{*} S_{0}+\sigma_{5}^{*}\right) t}\right)\right)^{-1} .
$$

A prediction interval of possible numbers that $I_{t}$ may take at the time $s$ with a confidence $\alpha$ is $\left[i_{L}, i_{U}\right]$ where $\left(i_{L}, i_{U}\right)$ is the optimal solution of the optimization problem

$$
\left\{\begin{array}{l}
\min _{i_{L}, i_{U}} i_{U}-i_{L} \\
\text { subject to: } \\
\quad \Phi_{S}\left(i_{U}\right)-\Phi_{s}\left(i_{L}\right) \geq \alpha \\
\quad i_{L} \text { and } i_{U} \text { are positive integers. }
\end{array}\right.
$$

Below are the details about the procedure to compute the prediction interval of active cases numbers based on the optimization model (19). For simplicity, we take the computation of prediction interval with confidence $\alpha=0.95$ on April 15 as an example.

Step 1 Set April 11, 2020 as the initial day, and set $I_{0}=1138$ in Eq. (18) which is the number of active cases on April 11, 2020. Then for April 15, i.e., the fourth day from the initial day, we have $t=4$.

Step 2 Set

$$
\beta_{I}^{*}=1.1745 \times 10^{-11}, \quad \sigma_{1}^{*}=7.7866 \times 10^{-12}, \quad \mu_{I}^{*}=0.0785, \quad \sigma_{5}^{*}=0.0296
$$

in Eq. (18) which have already been obtained in Sect. 3 based on the numbers of active cases and closed cases in Mainland China from February 12 to April 11, 2020. Then we get

$$
\Phi_{4}(x)=\left(1+\exp \left(\frac{\pi}{\sqrt{3}} \cdot \frac{6.7889-\ln x}{0.1619}\right)\right)^{-1} .
$$


Step 3 Set $\alpha=0.95$, and solve the optimization problem

$$
\left\{\begin{array}{l}
\min _{x_{1}>0, x_{2}>0} x_{2}-x_{1} \\
\text { subject to: } \\
\quad \Phi_{4}\left(x_{2}\right)-\Phi_{4}\left(x_{1}\right) \geq \alpha
\end{array}\right.
$$

by using the function "fmincon" in Matlab toolbox. The optimal solution is $\left(x_{1}, x_{2}\right)=(617.6,1199.6)$.

Step 4 Noting that $i_{L}$ and $i_{U}$ are positive integers in the optimization problem (19), we have $i_{L}=617$ and $i_{U}=1200$ as $\Phi_{4}(1200)-\Phi_{4}(618)<0.95$ and $\Phi_{4}(1199)-\Phi_{4}(617)<0.95$.

Hence, with $95 \%$ confidence, the number of active cases on April 15 will be no greater than 1200 and no less than 617.

\section{Conclusion}

By means of uncertain differential equations, an uncertain SEIAR model was derived to describe the spread of an epidemic. Specifically, with the COVID-19 cases data released by the National Health Commission of the People's Republic of China, the parameters in an uncertain epidemic model were estimated by following the method of moments. Furthermore, a method to predict the numbers of active cases was presented. Further research may consider the influence of vaccination to susceptible individuals, and design efficient vaccination strategies based on the uncertain SEIAR model.

Acknowledgements This research was supported by the Project of High-level Teachers in Beijing Municipal Universities in the Period of 13th Five-year Plan (No. CIT\&TCD20190338), the Humanity and Social Science Foundation of Ministry of Education of China (No. 19YJAZH005), the Young Academic Innovation Team of Capital University of Economics and Business (No. QNTD202002), and the special fund of basic scientific research business fees of Beijing Municipal University of Capital University of Economics and Business (No. XRZ2020016).

\section{References}

Artalejo, J. R., Economou, A., \& Lopez-Herrero, M. J. (2015). The stochastic SEIR model before extinction: Computational approaches. Applied Mathematics and Computation, 265, 1026-1043.

Chen, X., \& Liu, B. (2010). Existence and uniqueness theorem for uncertain differential equations. Fuzzy Optimization and Decision Making, 9(1), 69-81.

Chen, X., \& Gao, J. (2013). Uncertain term structure model of interest rate. Soft Computing, 17(4), 597-604.

Fang, J., Li, Z., Yang, F., \& Zhou, M. (2018). Solution and $\alpha$-path of uncertain SIS epidemic model with standard incidence and demography. Journal of Intelligent \& Fuzzy Systems, 35(1), 927-935.

Gao, R. (2016). Milne method for solving uncertain differential equations. Applied Mathematics and Computation, 274, 774-785.

Gray, A., Greenhalgh, D., Hu, L., Mao, X., \& Pan, J. (2011). A stochastic differential equation SIS epidemic model. SIAM Journal on Applied Mathematics, 71(3), 876-902.

Ji, C., Jiang, D., \& Shi, N. (2012). The behavior of an SIR epidemic model with stochastic perturbation. Stochastic Analysis and Applications, 30(5), 755-773.

Li, Z., Sheng, Y., Teng, Z., \& Miao, H. (2017). An uncertain differential equation for SIS epidemic model. Journal of Intelligent \& Fuzzy Systems, 33(4), 2317-2327. 
Li, Z., \& Teng, Z. (2019). Analysis of uncertain SIS epidemic model with nonlinear incidence and demography. Fuzzy Optimization and Decision Making, 18(4), 475-491.

Liu, B. (2007). Uncertainty Theory (2nd ed.). Berlin: Springer.

Liu, B. (2008). Fuzzy process, hybrid process and uncertain process. Journal of Uncertain Systems, 2(1), $3-16$.

Liu, B. (2009). Some research problems in uncertainty theory. Journal of Uncertain Systems, 3(1), 3-10.

Liu, Y., \& Liu, B. (2020). Estimating unknown parameters in uncertain differential equation by maximum likelihood estimation, Technical Report.

Liu, Z. (2020). Generalized moment estimation for uncertain differential equations, Technical Report.

Liu, Z., \& Yang, Y. (2020). Pharmacokinetic model based on multifactor uncertain differential equation, Technical Report.

National Health Commission of the People's Republic of China. (2020). Coronavirus disease (COVID-19) cases reports. http://www.nhc.gov.cn/xcs/yqtb/list_gzbd.shtml, Accessed April 12, 2020.

Sheng, Y., Gao, R., \& Zhang, Z. (2017). Uncertain population model with age-structure. Journal of Intelligent \& Fuzzy Systems, 33(2), 853-858.

Sheng, Y., Yao, K., \& Chen, X. (2020). Least squares estimation in uncertain differential equations. IEEE Transactions on Fuzzy Systems. https://doi.org/10.1109/TFUZZ.2019.2939984.

Yang, X., \& Gao, J. (2015). Linear-quadratic uncertain differential game with application to resource extraction problem. IEEE Transactions on Fuzzy Systems, 24(4), 819-826.

Yang, X., \& Ralescu, D. A. (2015). Adams method for solving uncertain differential equations. Applied Mathematics and Computation, 270, 993-1003.

Yao, K., \& Chen, X. (2013). A numerical method for solving uncertain differential equations. Journal of Intelligent \& Fuzzy Systems, 25(3), 825-832.

Yao, K., Gao, J., \& Gao, Y. (2013). Some stability theorems of uncertain differential equation. Fuzzy Optimization and Decision Making, 12(1), 3-13.

Yao, K., \& Liu, B. (2020). Parameter estimation in uncertain differential equations. Fuzzy Optimization and Decision Making, 19(1), 1-12.

Zhang, Y., Gao, J., \& Huang, Z. (2017). Hamming method for solving uncertain differential equations. Applied Mathematics and Computation, 313, 331-341.

Zhu, Y. (2010). Uncertain optimal control with application to a portfolio selection model. Cybernetics and Systems: An International Journal, 41(7), 535-547.

Publisher's Note Springer Nature remains neutral with regard to jurisdictional claims in published maps and institutional affiliations. 